\title{
The clinical comparison of Oxoid Signal with Bactec blood culture systems for the detection of streptococcal and anaerobic bacteraemias
}

\author{
NICOLETTE A. HUTCHINSON, F. D. THOMAS* and D. C. SHANSON \\ Department of Medical Microbiology, Charing Cross and Westminster Medical School, 17 Horseferry Road, \\ London SW1P 2AR and "Queen Elizabeth Military Hospital, Woolwich
}

\begin{abstract}
Summary. Blood cultures were taken from 47 patients 1-2 min after dental extraction. These samples were tested by the radiometric Bactec 460 and Oxoid Signal systems for the detection of streptococcal and anaerobic bacteraemias. Streptococci were isolated from $19(40 \%)$ patients and anaerobes from $15(32 \%)$. In this study the Oxoid Signal system was significantly better for isolating oral anaerobes than the Bactec system; five isolates were obtained with the Bactec system and 14 with the Signal system. There was no significant difference in the isolation of streptococci between these two systems (Bactec 14, Oxoid Signal 10). The contamination rate was $4.1 \%$ for Bactec and $7.5 \%$ for the Oxoid Signal system.
\end{abstract}

\section{Introduction}

Oxoid Signal ${ }^{\circledR}$ is a single bottle blood-culture system that uses a defined medium capable of supporting growth of both aerobic and anaerobic bacteria. Once a bottle has been inoculated and received in the laboratory, a clear plastic upper cylinder, the Signal device, is attached via its needle which is inserted through the bottle hub. Microbial growth generates gas that forces medium up the needle into the cylinder, thus signalling a positive culture.

Blood cultures are essential in the diagnosis of infective endocarditis; therefore, blood-culture systems must be effective in the prompt detection of viridans-type streptococci-the most common causative agents of endocarditis. In this study the Oxoid Signal blood culture system was compared with the radiometric Bactec $460^{\circledR}$ for the isolation of streptococci. The detection of anaerobic bacteraemia was also studied, as blood culture media vary greatly in their ability to support growth of inocula of nonsporing anaerobes. A number of previous studies by Shanson et al. ${ }^{1-3}$ showed that streptococci could be isolated from $25-47 \%$ of blood cultures taken within 2 min of dental extraction. For this reason we have investigated patients undergoing dental extraction as our source of blood cultures.

\section{Materials and methods}

\section{Blood culture media}

In this study the Bactec bottles used were the aerobic

Received 19 Sept. 1991 ; revised version accepted 18 March 1992.
6B and the anaerobic 7D, each containing $30 \mathrm{ml}$ of Soybean-caesul digest broth incorporating sodium polyanetholsuphonate $0.025 \%$. The Oxoid Signal bottle contained $80 \mathrm{ml}$ of Oxoid Special Broth with sodium polyanetholsuphonate $0.03 \%$.

\section{Blood collection methods}

Adults undergoing extraction of impacted wisdom teeth (third molars) under general anaesthesia at Queen Elizabeth Military Hospital, Woolwich were studied. Patients who had received antibiotics within the week before surgery were excluded. A volume (20 ml) of blood was drawn 1-2 min after extraction of the first tooth and, from this, samples were inoculated aseptically into each of the Bactec bottles $(5 \mathrm{ml})$ and into the Signal bottle $(10 \mathrm{ml})$. The order in which the bottles were inoculated was varied. The bottles were then transported at the end of the operation list to Westminster Hospital Microbiology Laboratory.

\section{Processing of blood cultures}

Upon arrival in the laboratory all cultures were processed immediately. The Bactec vials were incubated at $37^{\circ} \mathrm{C}$; the $6 \mathrm{~B}$ aerobic bottles were agitated for the first $48 \mathrm{~h}$. Both Bactec bottles were examined macroscopically and radiometrically twice on the first 2 days and once daily thereafter to day 7 . Gramstained films and subcultures were made from bottles giving a positive growth index, i.e., exceeding the aerobic and anaerobic predetermined thresholds of 30 and 25 respectively. Terminal subcultures were performed on those vials remaining negative after 7 days.

On receipt of the Oxoid Signal culture bottles, the 
Signal device was attached and the bottle was incubated at $37^{\circ} \mathrm{C}$ with agitation for the first $48 \mathrm{~h}$. The Signal bottles were examined for macroscopic evidence of growth and for a positive signal (fluid in the upper cylinder) twice daily for the first 2 days and once daily thereafter to day 7 . Any bottles that were positive by macroscopic examination or the Signal device were subcultured aerobically and anaerobically. Terminal subcultures were performed on those that remained negative after 7 days. All blood agar plates for anaerobic culture were incubated for 7 days in an anaerobic cabinet $\left(\mathrm{N}_{2} 80 \%, \mathrm{CO}_{2} 10 \%, \mathrm{H}_{2} 10 \%\right)$.

\section{Identification of isolates}

Suspected anaerobes were subcultured aerobically and anaerobically for up to 7 days. Isolates that grew only anaerobically and were sensitive to metronidazole were confirmed as anaerobes. These organisms were further identified by colonial morphology, reaction with Gram's stain and antibiotic sensitivity test with the anaerobic identification Mastring (Diamed Diagnostics) that includes penicillin 2 units, erythromycin $60 \mu \mathrm{g}$, rifampicin $15 \mu \mathrm{g}$, colistin $10 \mu \mathrm{g}$, kanamycin $1000 \mu \mathrm{g}$ and vancomycin $5 \mu \mathrm{g}$ disks.

Colonies from the aerobic plate were examined morphologically and by Gram's stain. Appropriate colonies that were coagulase- and catalase-negative were presumptively identified as streptococci. These were further identified by the API 20 Streptococcal identification test (bioMérieux UK Ltd, Basingstoke, Hants) and optochin sensitivity.

\section{Results}

Twenty-four strains of streptococci were obtained from $19(40 \%)$ blood culture sets; 15 were isolated from Bactec bottles and 12 from the Signal system (table I). There was no significant difference between the Signal and radiometric Bactec systems in their overall isolation of streptococci. The same streptococcal species was isolated with both systems on only three occasions.

Nineteen strains of anaerobes were isolated from 15 $(32 \%)$ samples, with the Bactec detecting five anaerobes and the Signal system 14 (table II). In 10 (21\%) samples an anaerobe was detected by the Signal alone; in contrast only one anaerobe was detected by the Bactec alone. Using the comparison of proportions, these results show that the Oxoid Signal system is significantly better at the $5 \%$ level in the recovery of non-sporing anaerobes than the radiometric Bactec system. In the three cases in which the same anaerobe was isolated by both systems there was no difference in the time taken to detect the pathogen.

In three cases the Oxoid Signal system showed macroscopic evidence of bacterial growth but the "Signal" was negative. These samples were also found
Table I. Isolation of streptococci from the two blood culture systems

\begin{tabular}{rll}
\hline Case no. & \multicolumn{1}{c}{ Bactec } & \multicolumn{1}{c}{ Signal } \\
\hline 1 & S. mitis & - \\
2 & S. sanguis + & \\
3 & Aerococcus viridans & S. sanguis \\
4 & S. milleri I & S. mitis I \\
5 & S. sanguis & S. mitis \\
6 & S. mitis & - \\
7 & S. milleri & S. milleri II \\
8 & S. milleri II & - \\
9 & S. milleri & S. mitis \\
10 & - & S. morbillorum \\
11 & S. morbillorum & - \\
12 & - & S. milleri I \\
13 & S. mitis & - \\
14 & S. sanguis II & S. mitis + S. sanguis \\
15 & S. milleri II & - \\
16 & $\overline{S . ~ s a n g u i s ~}$ & S. milleri + S. mitis \\
17 & S. sanguis & \\
19 & - &
\end{tabular}

Table II. Isolation of anaerobes from the two blood culture systems

\begin{tabular}{rll}
\hline Case no. & \multicolumn{1}{c}{ Bactec } & \multicolumn{1}{c}{ Signal } \\
\hline 1 & Fusobacterium sp. & $\begin{array}{l}\text { Fusobacterium } \text { sp. }+ \\
\text { anaerobic coccus } \\
\text { BPGNA + }\end{array}$ \\
2 & - & anaerobic coccus \\
3 & - & Fusobacterium sp. \\
4 & - & - \\
5 & Prevotella oralis BPGNA & \\
& Bacteroides ureolyticus & B. oralis \\
6 & (Actinomyces sp.) & Fusobacterium sp. \\
7 & - & Anaerobic coccus \\
9 & - & BPGNA \\
10 & - & BPGNA \\
11 & P. oralis & BPGNA \\
12 & - & Anaerobic coccus \\
13 & B. ureolyticus & B. ureolyticus \\
14 & B. & B. ureolyticus \\
15 & Anaerobic coccus & Anaerobic coccus \\
\hline
\end{tabular}

BPGNA, black-pigmented gram-negative anaerobe.

to be leaking. One false negative result, i.e., growth only on terminal subculture, occurred with both each system. The contamination rates for both systems were higher than in earlier studies ${ }^{4-6}$ with a rate of $4 \%$ for the Bactec and $7.5 \%$ for the Oxoid Signal.

\section{Discussion}

The Oxoid Signal system was significantly better for detecting oral anaerobes than the radiometric Bactec 460. This has not been noted in earlier trials. ${ }^{4-6}$ Previous studies comparing Oxoid Signal with radiometric Bactec showed that without agitation the Oxoid Signal was significantly poorer for detecting streptococci, ${ }^{4}$ but with agitation this difference disappeared. 
Our study confirms these findings, showing no significant difference between the two systems for the detection of viridans-type streptococci. However, the greater number of streptococcal isolates was noted with the Bactec system and it is possible that a much larger study might have shown a significant difference between the two systems.

The Oxoid Signal blood culture system employs a single bottle and has the advantage of not requiring expensive equipment. Our trial showed that the Oxoid Signal gave similar results to the radiometric Bactec 460 for the detection of viridans-type streptococci but was significantly better in the recovery of oral anaerobes.

\section{References}

1. Shanson DC, Thomas FD, Johnstone D. Improving detection of 'viridans streptococcus' bacteraemia by adding sodium polyanethol sulphonate to blood cultures. J Clin Pathol 1985; 38: 1346-1348.

2. Shanson DC, Akash S, Harris M. Clinical comparison of glucose broth with nutrient broth blood cultures for the detection of "Streptococcus viridans" bacteraemia. J Med Microbiol 1983; 16: 497-499.

3. Shanson, DC, Moule I, Tadayon M. Clinical comparison of anaerobic blood-culture media for detecting bacteraemia due to viridans streptococci and oral anaerobes. $\mathrm{J} \mathrm{Med}$ Microbiol 1985; 19: 187-193.
There are two main alternative explanations for the superior results for the detection of anaerobes by the Signal system: (a) difference in media composition and (b) different blood volumes. The medium in the Signal system includes a type of thioglycollate broth that could be more effective than the medium used in the anaerobic Bactec bottle. Alternatively the difference may be explained by the fact that the whole $10-\mathrm{ml}$ sample of blood is examined for the presence of anaerobes by the Signal bottle whereas only half the sample $(5 \mathrm{ml})$ is investigated for anaerobes by the two bottle Bactec system.

\footnotetext{
We are most grateful to Mrs M. Mountain for her secretarial
} help.

4. Weinstein MP, Mirrett S, Reller LB. Comparative evaluation of Oxoid Signal and BACTEC radiometric blood culture systems for the detection of bacteraemia and fungemia. $J$ Clin Microbiol 1988; 26: 962-964.

5. King A, Bone G, Phillips I. Comparison of radiometric and gas capture system for blood cultures. J Clin Pathol 1986; 39 : 661-665.

6. Weinstein MP, Mirrett S, Reimer LG, Reller LB. Effect of agitation and terminal subcultures on yield and speed of detection of the Oxoid Signal Blood Culture System versus the BACTEC radiometric system. J Clin Microbiol 1989; 27: $427-430$. 